\title{
Leben mit der Mauer
}

\author{
Reiner Kunzes Schießbefehl
}

\section{Steffi Retzlaff}

\begin{abstract}
Zusammenfassung
Das Leben in der DDR, mit einer Mauer, die das Land viele Jahre lang von seinem westdeutschen Teil trennte, ist vielen Lerner/innen auf den ersten Blick unzugänglich. Durch einen dramapädagogischen Ansatz können Lerner/innen Möglichkeiten gegeben werden, sich mit gewissen Rollen und Personen zu identifizieren und fiktive Situationen zu schaffen, die nicht unmittelbar die eigenen Erfahrungen und Lebensräume reflektieren. Ein kulturell fremder Text wird körperlich-sinnlich begreifbar gemacht und stellt ein Deutungsangebot für eigene Erlebnisse und Fantasien dar. In der folgenden Beschreibung wird eine exemplarische Unterrichtseinheit dargestellt, die das Thema 'Leben mit der Mauer' anhand von Improvisationsübungen und szenischen Interpretationen zu Reiner Kunzes Kurzprosatext Schießbefehl dramapädagogisch aufarbeitet.
\end{abstract}

\section{Einführung}

Schulische und akademische Einrichtungen sind neben der Familie und dem Freundeskreis der entscheidende Ort, an dem Kinder, Jugendliche und junge Erwachsene ihre Identität entwickeln. Die Schule oder Universität sollte sich daher nicht nur um die schulische oder akademische, was ja meistens kognitive Bildung bedeutet, sondern auch um die Persönlichkeitsentwicklung der Lerner/innen kümmern. Dieser Aspekt wird in vielen Ländern ignoriert; Schulen, Schulministerien und Universitäten folgen oft einem Erziehungsansatz, der sich auf den Kopf konzentriert, Hand, Fuß und Herz jedoch ignoriert. Durch einen ganzheitlichen Ansatz beim Lehren und Lernen kann Unterricht als geistiges, emotionales, spirituelles und körperliches Ereignis begreifbar gemacht werden. Mahatma Gandhi bietet eine überzeugende Definition von holistischer Erziehung an:

I hold that true education of the intellect can only come through a proper exercise and training of the bodily organs, e.g., hands, feet, eyes, ears, nose, etc. In other words an intelligent use of the bodily organs in a child provides the best and quickest way of developing his intellect. But unless the development of the mind and body goes hand in hand with a 
corresponding awakening of the soul, the former alone would prove to be a poor lopsided affair. By spiritual training I mean education of the heart. A proper and all round development of the mind, therefore, can take place only when it proceeds pari passu with the education of the physical and spiritual faculties of the child. They constitute an indivisible whole. (Gandhi 1958: 151)

Manfred Schewes (1993) ganzheitlich-handlungsorientierter Ansatz beschäftigt sich mit der Verbindung von Drama/Theater und der Fremdsprachenvermittlung, d.h. mit der unterrichtlichen Inszenierung von Fremdsprache. Die Grundidee hierbei ist, dass Fremdsprachenlehrer/innen viel von professionellen Theatermachern lernen können (cf. Schewe 2000: 72). Die szenische Improvisationsarbeit steht im drampädagogischen Unterricht im Mittelpunkt:

Die Gestaltung von szenischen Improvisationen geht einher mit einem körperbezogenen, auf das Verstehen und Anwenden von fremder Sprache, Literatur und Kultur gerichteten Tun von Lernenenden und Lehrenden; es ist gekoppelt an Erfahrungen, die gemacht werden, wenn solche Improvisationen vorbereitet, durchgeführt und ausgewertet werden (Schewe 2000: 72).

Ingo Schellers Konzept des szenischen Spiels und der szenischen Interpretation ist ebenfalls text- und handlungsbezogen, subjekt- und gruppenbezogen und integriert die Erfahrungen der Lerner/innen. Im Kern bieten diese Verfahren (in der Auseinandersetzung mit einem Text) die Gelegenheit, Vorstellungen, Projektionen und Bedeutungen zu vergegenständlichen und damit bewusst zu machen, so dass „auch vergessene oder abgewehrte, asoziale Erlebnisse, Phantasien und Wünsche (wieder)entdeckt und überdacht werden" können (Scheller 1999: 320).

Im Folgenden wird eine mehrstündige dramapädagogische Unterrichtseinheit zum Thema 'Leben mit der Mauer' beschrieben. Mein Anspruch an diese Unterrichtseinheit ist, den Lernern und Lernerinnen einen handlungs- und erfahrungsbezogenen Deutsch als Fremdsprachenunterricht zu ermöglichen, obgleich es bei diesem Thema um kulturelle, historische und sozio-politische Erfahrungen geht, die nicht unmittelbar die der Lerner/innen reflektieren.

Der Text, der mit und für die Lerner/innen erfahrbar gemacht wird, heißt Schießbefehl aus Reiner Kunzes Kurzprosaband Die wunderbaren Jahre. Das Niveau der Lerner/innen ist zwischen B2 und C1. ${ }^{1}$ (Ich habe diese Einheit aber auch schon mit Lerner/innen auf dem B1 Niveau ohne Probleme durchgeführt und außerdem als Lehrer/innenfortbildung für DaF und ESL veranstaltet.) Die Zusammensetzung der Lerngruppe ist sehr heterogen: Schüler/innen, Student/innen, erwachsene 'Arbeitnehmer/innen' und Rentner/innen. Aufgrund dieser Tatsache ist auch das Vorwissen und Interesse der Lerner/innen zum Thema DDR, Mauer und Schießbefehl sehr unterschiedlich.

\footnotetext{
${ }^{1}$ Ich benutze die Kennzeichnungen B1, B2 und C1 auf der Grundlage des Gemeinsamen europäischen Referenzrahmen für Sprachen: Lernen, lehren, beurteilen. http://www.goethe. de/ z/50/commeuro/deindex.htm.
} 
Kunzes Schießbefehl-Einheit benötigt in ihrer Gesamtheit ca. 4-5 Unterrichtsstunden (45-50 Minuten pro Stunde). Die Allgemeinziele beinhalten eine Rollenbiographie schreiben (spontanes) Sprechen und die Vertiefung von Hör-, Lese- und Textverständnis. Im Speziellen zielt diese Unterrichtseinheit darauf $\mathrm{ab}$, die Lerner/innen für das Thema 'Leben mit der Mauer' und für einige Besonderheiten des DDR-Systems zu sensibilisieren. Im Rahmen dieser Einheit erwerben die Lerner/innen linguistisches und zugleich sozio-politisches und historisches Fachwissen zu den Themen 'Berliner Mauer' und 'Leben in der DDR'. Des Weiteren sollen die Lerner/innen in der Auseinandersetzung mit diesen Themen durch Mittel des Theaters und der szenischen Interpretation ihre Kreativität, Empathie sowie soziale und emotionale Intelligenzen entdecken und schulen.

Die folgende Beschreibung der Unterrichtseinheit gliedert sich in zwei Teile: Eine dramapädagogische Annäherung an das Thema 'Leben mit der Mauer' und eine literaturbasierte Auseinandersetzung mit dem Thema 'Leben mit der Mauer'. Zunächst jedoch gibt es einige kurze biographische Informationen über Reiner Kunze.

Reiner Kunze wurde 1933 in Oelsnitz als Sohn eines Bergarbeiters geboren. Er studierte Journalistik und Philosophie an der Universität Leipzig, an der er auch von 1955-1959 arbeitete. Nach seiner Entlassung vom Universitätsdienst (aus politischen Gründen), verdiente er sein Geld zunächst als Hilfsschlosser. Seit 1962 ist Reiner Kunze als freiberuflicher Schriftsteller tätig. 1968 trat Kunze aus Protest gegen den Einmarsch der Warschauer-Pakt-Staaten in die Tschechoslowakei aus der Sozialistischen Einheitspartei Deutschlands (SED) aus. Die Veröffentlichung seines Prosabandes Die wunderbaren Jahre ${ }^{2}$ in einem westdeutschen Verlag 1976 führte zum endgültigen Bruch mit dem DDRRegime. In diesem Werk beschreibt Kunze Momentaufnahmen aus dem Alltag der DDR-Jugend, die zu Anpassung und Gehorsam erzogen wird. Der Band ist in vier Themenkomplexe gebündelt (Friedenskinder, Federn, Verteidigung einer unmöglichen Metapher und Café Slavia) mit jeweils kurzen Texten über Szenen aus der inneren Wirklichkeit der DDR. Daraufhin wurde Kunze aus dem DDR-Schriftstellerverband ausgeschlossen, was so viel wie Berufsverbot bedeutete. 1976 schloss er sich dem Protest gegen die Ausbürgerung des Liedermachers Wolf Biermann an und siedelte 1977 mit seiner Familie von der DDR in die BRD über. Seitdem lebt er in der Nähe von Passau. Für seine Werke erhielt Kunze zahlreiche in- und ausländische Literaturpreise. Seine Lyrik und Prosa wurden in dreißig Sprachen übersetzt.

\footnotetext{
${ }^{2}$ Der Titel Die wunderbaren Jahre ist ein Zitat aus der Grasharfe von Truman Capote: „Ich war elf, und später wurde ich sechzehn. Verdienste erwarb ich mir keine, aber das waren die wunderbaren Jahre." Kunze beginnt mit diesem Zitat den Abschnitt Verteidigung einer unmöglichen Metapher.
} 


\section{Dramapädagogische Annäherung an das Thema 'Leben mit der Mauer'}

Am 13. August 1961 wurde in Berlin mit dem Bau der Mauer zwischen Ost und West begonnen. Stacheldraht, eine Betonmauer, Elektrozäune, Wachtürme und Wachposten sowie Selbstschussanlagen und Minenfelder entlang der Grenze trennten die zwei Teile Deutschlands von da an für mehr als 28 Jahre. Am 9. November 1989 fiel die Mauer. Die Geschichte der DDR ist noch längst nicht zu Ende erzählt und vielen der heutigen Jugendlichen und auch erwachsenen Lerner/innen nicht unbedingt zugänglich. Ein dramapädagogischer Ansatz, wie in den folgenden Erklärungen exemplarisch beschrieben, kann helfen, das Thema 'Leben mit der Mauer' erfahrbar zu machen. ${ }^{3}$

\subsection{Einstieg/Aufwärmen/Energetisieren}

Ein 'warm-up' sollte zu Beginn jeder Arbeitseinheit durchgeführt werden. Sich aufwärmen ist ein Sich-Bereitmachen für das Spiel, ein „Sich-Finden, Sich-Einfinden: in der Gruppe auch ein gemeinsamer Start vom 'ich' zum 'wir'" (Mettenberger 2004: 10). Bei der Theaterarbeit wird es 'körperlich', das ist ein Grund, warum Aufwärmen und Energetisieren sehr wichtig sind. Warm-ups haben daneben aber auch noch andere Funktionen, z.B. Kennenlernen, Körperund Bewegungswahrnehmung, Konzentrationssteigerung oder Sensibilisierung für das zu bearbeitende Thema. Wichtig ist dabei, die Aufwärmübungen so einzusetzen, dass sie auf die nachfolgenden Schritte abgestimmt sind, diese sozusagen schon 'vorpfaden'. Zunächst sollen die Lerner/innen energetisiert werden. Dazu eignen sich Übungen, die den Körper und Geist wach machen wie beispielsweise Laufen oder Joggen im Raum mit verschiedenen Geschwindigkeiten (1-10 wobei 1 Stillstand bedeutet und 10 das schnellste Tempo ist) und gymnastische Streckübungen, Yoga oder Tai-Chi. Bei der Streckübung 'Apfelpflücken' stehen die Lerner/innen im Kreis und strecken sich, um an die Äpfel, die am Baum hängen heranzukommen. 'Apfelpflücken' bereitet gleichzeitig auf den nächsten Schritt vor, nämlich sich zu strecken, um über eine imaginäre Mauer zu schauen.

Die Lehrperson demonstriert nun, wie sie sich streckt, um über eine Mauer zu schauen. Dabei beobachtet sie etwas, das sie der Gruppe in entsprechender Stimmlage, also 'atmosphärisch' mitteilt, z.B.:

- „Oh wie süß, da spielen die Kinder wieder.“

- „Mensch, dass ist doch heiß, warum bekommt der Hund nichts zu trinken?!“

\footnotetext{
${ }^{3}$ Die in diesem Abschnitt beschriebene Vorgehensweise wurde von Manfred Schewe entwickelt und von ihm als Seminarleiter im Rahmen der zweiwöchigen Fortbildung 'Theater im Unterricht' am Goethe-Institut Berlin (Sommer 2009) demonstriert. Die in Abschnitt 3 vorgestellten Verfahren sind Weiterentwicklungen aus meiner Unterrichtspraxis.
} 
- „Pass auf! Der hat ein Gewehr!“

Nacheinander erzählt nun jede/r, was sie/er hinter der Mauer sieht. Auf diese Weise entstehen diverse 'Mauer-Kontexte'; die Lerner/innen werden für das zu bearbeitende Thema sensibilisiert.

\subsection{Touristenführung und Assoziation (Geste)}

Durch die Improvisationsübung 'Touristenführung' werden das Hintergrundwissen und die Multikulturalität einer Lerngruppe bewusst aktiviert/genutzt. Die Lehrperson gibt die folgende Anweisung: „Du führst eine Gruppe von Touristen an die Berliner Mauer und erklärst dieser Gruppe in lebendiger Weise, was du über die Mauer weißt." In vielen Lerngruppen gibt es verschiedene Nationalitäten und Muttersprachen, das kann man sich hier von Nutzen machen, indem man die Lerner/innen in ihrer eigenen Sprache die Touristenführung machen lässt (wie im wirklichen Berlin!). Das Thema 'Berliner Mauer' ist durch die Touristenführung eingeführt und erfährt nun eine erste Erweiterung: Die Lerner/innen überlegen sich nun eine Geste oder Bewegung, die ihnen zum Thema einfällt, und erproben diese für sich im Raum (z.B. Hände am Hals, als ob man erstickt/erwürgt wird, Gewehr in der Hand, am Boden liegend). Gestik und Gang sind unmittelbarer Ausdruck unseres Körpers, der wiederum Sprachrohr für (phantasierte) Haltungen und damit verbundene Empfindungen ist. Diese Übung sensibilisiert für die Wirkung von Gesten und Bewegungen und die dadurch wach gerufenen (körperlichen) Vorstellungen von der Berliner Mauer.

\subsection{Verkörperung der Mauer}

Diese letzte Improvisationsübung im Teil 'Dramapädagogische Annäherung an das Thema 'Leben mit der Mauer" hilft, Ereignisse an der Mauer in derVorstellung der Lernenden zu inszenieren. Gleichzeitig ist es eine Übung, die grammatische Strukturen festigt. Die Lehrperson demonstriert, wo im Raum sich die Mauer befindet, und erklärt, dass die Lerner/innen nun eine Gruppenskulptur zum Thema 'Mauer' bauen. Wichtig bei diesem theaterästhetischen Mittel ist die genaue Anweisung: Die Gruppenskulptur entsteht nach und nach (Vermeidung von Gleichzeitigkeit!). Die Lerner/innen gehen nacheinander in das Bild, wobei sie ihre Geste und Bewegung aus dem vorherigen Arbeitsschritt 'groß machen'. Sie positionieren sich entweder in Blickrichtung Osten oder in Blickrichtung Westen. Zusätzlich kann man am Ende, wenn alle in der Gruppenskulptur stehen, noch eine Stimmen- oder Geräuschcollage machen. Die Lerner/innen werden gebeten, ein Geräusch zu machen, das sie mit der Mauer oder ihrer Geste assoziieren. Auf ein Signal der Lehrperson hin machen alle ihre Geräusche gleichzeitig. Man kann die Lerner/innen auch bitten, die Augen dabei zu schließen und die so entstehende Collage zu fühlen. Die Darstellung der Mauer durch Geräusche, Stimmen und Töne sensibilisiert für die Emotionalität des 
Themas und des zu lesenden Textes von Reiner Kunze. Die Geräuschcollage wurde von den meisten Lerner/innen als 'Gänsehaut-Moment' empfunden.

Nach diesem 'symbolischen' Zugang durch die Gruppenskulptur werden die Lerner/innen in einem letzten Schritt aufgefordert, sich vorzustellen, ein Stein in der Berliner Mauer zu sein. Die Lehrperson verweist darauf, dass die Mauer 28 Jahre lang existierte und in dieser Zeit viel geschehen ist. Die Lerner/innen werden zum Stein und berichten aus dieser Perspektive, was sie gerade sehen oder gesehen haben. Die Lehrperson tippt den Lerner/innen nacheinander auf die Schulter und der/diejenige spricht seinen/ihren Satz. Hier kann man verschiedene Grammatikstrukturen wiederholen und festigen. Die Lehrperson kann ihre Frage entweder im Präsens oder im Perfekt stellen: Was siehst/fühlst du gerade? Was hast du gesehen/gefühlt? Die Lernenden antworten in der jeweiligen Zeitform.

Um den Lerner/innen Sprechimpulse zu geben, legt der/die Lehrer/in Bilder auf den Boden um die 'Mauer'. Diese Bilder müssen groß genug sein, dass man sie aus dem Stand sehen kann. Sie zeigen Einblicke in das Leben auf der Ostseite/DDR und der Westseite/BRD, z.B. bemalte Mauer und spielende Kinder vor der Mauer im Westen, Besuch von Kennedy und Reagan im Westen oder auf der Ostseite Bilder von Fluchtversuchen, bewaffneten Soldaten, Trabis aber auch von Liebespaaren oder lachenden Kindern. Abschließend sollte die Lehrperson noch eine Reflexionsrunde, ein Feedback über die bisherigen Schritte initiieren (s. auch Feedback am Ende der Gesamteinheit).

\section{Szenische Interpretation zum Thema 'Leben mit der Mauer'}

Ein handlungs- und erfahrungsbezogener Deutsch als Fremdsprache-Unterricht ist auch möglich, wenn es um kulturelle, historische und sozio-politische Erfahrungen geht, die nicht unmittelbar die eigenen reflektieren. Das Leben in der DDR mit einer Mauer ist vielen auf den ersten Blick unzugänglich. Wenn man Literatur wie Kunzes Schießbefehl jedoch dramapädagogisch aufarbeitet und den Lerner/innen somit Möglichkeiten gibt, sich zu identifizieren und fiktive Situationen zu schaffen, kann auch dieser Text körperlich-sinnlich begreifbar gemacht werden und ein Deutungsangebot für eigene Erlebnisse und Fantasien darstellen. In der dramapädagogischen Auseinandersetzung mit dem Text Schießbefehl fühlen sich die Lerner/innen in verschiedene Figuren ein und machen auf diese Weise im Schutzraum der Fiktion Erfahrungen, die ihnen im wirklichen Leben unmöglich wären: Sie übernehmen Rollen von DDR Polizist/innen und Stasi-Mitarbeiter/innen, von einem Jugendlichen aus der DDR, der eine Republikflucht plant, von einer Mutter, die ihren Sohn verliert. In den folgenden Ausführungen wird eine Annäherung an das Thema 'Leben mit der Mauer' mit den Mitteln der szenischen Interpretation exemplarisch dargestellt. Der Grundlagentext ist Reiner Kunzes Schießbefehl aus der Kurzprosasammlung Die wunderbaren Jahre (s. Anhang A). 


\subsection{Sprechhaltungen, Textschnipsel und Textlektüre (Zeitdauer insgesamt ca. 55-60 Minuten)}

Sprechhaltungen sammeln (ca. 3-4 Minuten) —Durch die im ersten Teil beschriebenen szenisch-improvisorischen Arbeitsschritte haben die Lerner/innen ihr (Vor)wissen zum Thema 'Mauer' aktiviert. In einem nächsten Schritt werden verschiedene Sprechhaltungen an der Tafel gesammelt (z.B.: laut, leise, schnell, flüsternd, schreiend, weinend, aufgeregt, gelangweilt, fordernd, schüchtern... ). Es ist neben der Vorbereitung für die nächste Aktivität gleichzeitig Vokabeltraining und Wiederholung von Adverbien. Damit später bestimmte Situationsund Rollenvorgaben szenisch umgesetzt werden können (z.B. beim Bau von Standbildern oder auf dem heißen Stuhl), sollten unterschiedliche Aspekte und Perspektiven von Figuren und Szenen wahrgenommen oder zumindest vorgestellt werden können.

Über Sprechübungen verschiedene Sprechhaltungen aktivieren (ca. 10 Minuten) Um die Lerner/innen für verschiedene Aspekte von Figuren zu sensibilisieren, werden nun über Sprechübungen verschiedene Sprechhaltungen aktiviert und ausprobiert. Scheller (1998: 92) merkt dazu an: „Das Experimentieren mit Intonationsmöglichkeiten, Brecht spricht vom 'Kneten' des Textes, aktiviert das sprachliche Verhaltensspektrum der Teilnehmer und bringt eine Vielfalt von Bedeutungsmöglichkeiten ans Tageslicht, die beim stillen Lesen selten zum Tragen kommen." Dazu verteilt die Lehrperson verschiedene Textschnipsel mit Sätzen aus dem Text Schießbefehl (s. Anhang B). Es ist ratsam, einiges an Wortschatz aus dem Text zu klären, bevor man mit den Sprechhaltungen beginnt:

- erwischen, gestehen, einrücken, erhängen, jmd. etwas antun, aushändigen, die Urne

- Facharbeiter mit Abitur ${ }^{4}$

Nun bekommt jede/r Lerner/in einen der Textschnipsel. Diese werden nacheinander im Kreis vorgelesen. Dann fordert die Lehrperson die Lerner/innen auf, durch den Raum zu gehen und ihren Satz/Textschnipsel in unterschiedlichen Sprechhaltungen und Intonationsvarianten auszuprobieren. Vielen Lerner/innen (und auch Lehrer/innen!) fällt es schwer, mit Sprechhaltungen und Lautstärken vor anderen zu experimentieren, daher bietet es sich an, dass man durch den Raum geht und sich bewegt, was wiederum auch das bewusste Atmen unterstützt. Im Sitzen ist die Atmung eingeschränkt. Wirksam ist auch, Musik im Hintergrund zu spielen. Als Alternative kann die Lehrperson eine bestimmte Sprechhaltung oder auch ein Gefühl für jede Runde vorgeben, z.B.: „Sprecht Euren Satz ganz traurig/Ihr seid ganz traurig.“ Nach ein paar Wiederholungen kommt die nächste Anweisung: „Schreit den Satz/Ihr seid

\footnotetext{
${ }^{4}$ Besonderheit des DDR-Bildungswesens: Man konnte gleichzeitig das Abitur und eine Berufsausbildung in 3 Jahren machen. Das 'normale' Abitur dauerte 2 Jahre.
} 
total wütend“, danach bittet man die Lerner/innen, ihren Satz ganz schüchtern zu sprechen etc.

Sprechhaltungen und Verhörsituation (ca. 5-7 Minuten) — Nachdem sich die Lerner/innen mit ihren Sätzen vertraut gemacht haben, wird ein Stuhl in die Mitte gestellt und die Lerner/innen stehen im Kreis darum. Eine Person beginnt, setzt sich auf den Stuhl und liest ihren Satz in einer 'normalen' Sprechhaltung vor. Die anderen nehmen diesen Satz auf und bieten ihn in einer anderen Sprechhaltung (leise, aggressiv, schreiend, flüsternd etc.) der Person auf dem Stuhl an und gehen dabei auf sie zu (Achtung: nicht gleichzeitig, sondern immer einer nach dem anderen). Danach setzt sich die nächste Person auf den Stuhl..... die nächste Person....etc. So entsteht eine Verhörsituation, die einen emotionalen Zugang zum Text schafft und eventuelle weitere Schritte in der Arbeit mit dem Text vorbereitet (z.B. Interview zwischen Gerhard und der Polizei/Stasi).

Diese Übung dient erstrangig der Erkundung der Wirkung von Sprechhaltungen, führt aber gleichzeitig auch in den Lesetext ein, sensibilisiert die Lerner/innen für die Ereignisse in der Geschichte (beispielsweise durch Sätze wie diese: „da kommen die und sagen, er hat über die Grenze gewollt“ oder „sie haben ihn erwischt" oder „er hat sich erhängt“).

Rekonstruktion des Textes (ca. 15 Minuten) - In einem letzten Schritt, bevor man die Kurzgeschichte zum erstmaligen Lesen austeilt, versuchen die Lerner/innen, in Gruppen die Reihenfolge der darin geschilderten Ereignisse mithilfe der Textschnipsel zu rekonstruieren. In den Gruppen wird dann darüber gesprochen, was passiert sein könnte. Danach werden die jeweiligen Versionen im Plenum verglichen und gemeinsam reflektiert.

Erste Textlektüre ohne Titel und mit Lückentextübung (ca. 20 Minuten) — Nun wird der gesamte Text ohne Titel zur ersten stillen Lektüre ausgegeben. Der Titel der Geschichte - Schießbefehl - würde die Lerner/innen in eine bestimmte (Interpretations-) Richtung führen, die zu diesem Zeitpunkt noch nicht erwünschtist. DieVorstellungskraft und Deutungsbreite soll für dieLerner/innen möglichst nicht eingeschränkt werden. Zur Orientierung stellt die Lehrperson W-Fragen: Wer? Wo? Was? Wann? Wie? Dabei merkt man schnell, dass es den Lerner/innen oft nicht klar ist, wer wer im Text ist. Das liegt daran, dass der grammatische Stil des Textes nicht ganz eindeutig ist mit Bezug auf die Deixis. Hier hilft eine Lückentextübung, um die Personalpronomen und Referenzpersonen im Text (Mutter, Sohn Gerhard, Polizei „Von hier“, Polizei/Stasi in P...) zu identifizieren (s. Anhang C). Diese Übung kann in Partner- oder Gruppenarbeit oder auch gemeinsam mit der gesamten Klasse durchgeführt werden. Danach wird der Text noch einmal individuell von den Lerner/innen gelesen und eventuelle Wortschatzfragen werden geklärt. 


\subsection{Standbilder bauen (Zeitdauer insgesamt ca. 45 Minuten)}

Die Methoden der szenischen Interpretation, z.B. Standbilder (Scheller 1989, 1998, 2004), eignen sich hervorragend für alle Lernstufen, da es darum geht Ereignisse, Situationen und Haltungen 'körperlich' und mimisch sichtbar zu machen. Das szenische Spiel erlaubt den Lerner/innen den Text nach ihren Vorstellungen, Ideen und Erfahrungen zu interpretieren. Laut Scheller gelingt es den wenigsten Leser/innen sich bei der bloßen Lektüre eines Textes 'einzufühlen'. Die Einfühlung, also das Thematisieren und Bewusstmachen eigener Erfahrungen und Fantasien, ist ein Schwerpunkt der szenischen Interpretation.

Standbilder sind bildliche Darstellungen, „fotografische Momentaufnahmen“, in denen „Szenen und Handlungsverläufe fixiert und Zustände, Haltungen und Beziehungen ausgestellt und gedeutet werden" (Scheller 1989: 62). Bei Standbildern werden Körperhaltungen, Gestik und Mimik 'eingefroren', um diese Momentaufnahme dann in einem zweiten Schritt zu interpretieren. Schewe (2000: 88) kommentiert die Arbeit mit Standbildern wie folgt:

Beim Planen, Proben und Präsentieren von Standbildern werden vielfältige sprachliche Lernprozesse in Gang gesetzt: Die KT sprechen sich $\mathrm{ab}$, machen Vorschläge, kritisieren, nehmen Stellung zur Wirkung eines Standbildes und interpretieren Standbilddetails; sie sprechen aus, was die Figuren im Standbild in dem gezeigten Moment denken oder sagen, oder nutzen das Standbild als Impuls, um einen Dialog, eine Geschichte, ein Szenario zu schreiben.

Die Arbeit mit Standbildern ist ein handlungsorientiertes Verfahren, d.h. es wird ein sicht- und fühlbares Produkt erstellt. Standbilder sind schnell zu erlernen und das Bauen von Standbildern ist leicht, erfordert aber etwas Disziplin und Präzision.

Die Methode des Standbildbauens (ca. 8-10 Minuten) — Zuerst muss man erläutern, wie ein Standbild gebaut wird. Am besten funktioniert das, indem man die Technik des Standbild-Bauens einmal langsam demonstriert und genau kommentiert, was man gerade macht. Die Regeln fürs Standbild-Bauen sollten schriftlich festgehalten werden, so dass die Lernenden immer wieder darauf zurückgreifen können. Am besten hat die Lehrperson diesen schriftlichen Teil schon vorab vorbereitet:

- Beim Bauen wird nicht gesprochen.

- Der/die Standbildbauer/in bringt die andere(n) Mitspieler/innen in die gewünschte Position und formt Körperhaltung und Gestik so lange, bis sie dem vorgestellten Bild entsprechen.

- Der/die Standbildbauer/in gibt den gewünschten Gesichtsausdruck vor, den die Mitspieler/innen imitieren. 
- Wenn alle Gestik und Mimik fertig geformt ist und das entstandene Bild der Vorstellung des/der Standbildbauer/in entspricht, wird es 'eingefroren'.

- Die Mitspieler/innen halten ihre Position 1-2 Minuten.

- Am Ende wird das Standbild gedeutet.

Standbilder entwickeln für Reiner Kunzes Schießbefehl (ca. 35 Minuten) — Für den Text von Reiner Kunze können Standbilder eingesetzt werden, um Spannungsmomente und Beziehungskonstellationen darzustellen, den gesamten Handlungsverlauf nachzuvollziehen oder auch abstrakte Begriffe und Situationen wie Angst, Verzweiflung oder Liebe zu visualisieren. Für die Interpretation des Textes Schießbefehl mithilfe von Standbildern werden die Lerner/innen in 6 Gruppen (s. Tabelle) eingeteilt. Wenn man nicht so viele Lerner/innen in der Klasse hat, dann baut jede Gruppe jeweils zwei Standbilder. Jede Gruppe bekommt einen bzw. zwei Abschnitt(e) des Textes zugeteilt. Die Gruppen sollen dann dafür Standbilder entwickeln. Bevor die einzelnen Gruppen ihre Standbilder bauen, weist die Lehrperson noch einmal auf die Regeln hin. Außerdem sollen die Lerner/innen folgendes in ihren Gruppen besprechen und beim Bauen der Standbilder beachten: Um welche Situation geht es? Was geschieht? Wo und wann genau findet diese Szene statt? Welche Gedanken und Gefühle haben die Personen gerade?

\begin{tabular}{|c|c|}
\hline Was? & Wie? \\
\hline $\begin{array}{l}\text { Gruppe 1: Anfang der } \\
\text { Geschichte/Abschied Mutter und Sohn } \\
\text { kurz vor der Abreise des Sohnes zum } \\
\text { Vater. }\end{array}$ & $\begin{array}{l}\text { Drei Lerner/innen: }{ }^{a} \text { Standbildbauer/in, } \\
\text { Mutter, Sohn }\end{array}$ \\
\hline $\begin{array}{l}\text { Gruppe 2: Gerhard wird erwischt } \\
\text { Gerhard an der Grenze. }\end{array}$ & $\begin{array}{l}\text { Vier Lerner/innen: Standbildbauer/in, } \\
\text { Sohn, } 2 \text { Grenzsoldaten }\end{array}$ \\
\hline $\begin{array}{l}\text { Gruppe 3: Vorbereitung auf } \\
\text { Gefängnisbesuch Mutter backt einen } \\
\text { Kuchen. }\end{array}$ & $\begin{array}{l}\text { Zwei Lerner/innen: Standbildbauer/in, } \\
\text { Mutter }\end{array}$ \\
\hline $\begin{array}{l}\text { Gruppe 4: Im Gefängnis/in der Zelle } \\
\text { Gerhard allein in seiner Gefängniszelle. }\end{array}$ & $\begin{array}{l}\text { Zwei Lerner/innen: Standbildbauer/in, } \\
\text { Sohn }\end{array}$ \\
\hline $\begin{array}{l}\text { Gruppe 5: Tödliche Nachricht Mutter } \\
\text { erhält die Nachricht, dass sich Gerhard } \\
\text { umgebracht hat. }\end{array}$ & $\begin{array}{l}\text { Vier Lerner/innen: Standbildbauer/in, } \\
\text { Mutter, } 2 \text { Stasi-Mitarbeiter/Polizisten }\end{array}$ \\
\hline $\begin{array}{l}\text { Gruppe 6: Die Urne Die Mutter bekommt } \\
\text { die Urne ausgehändigt. }\end{array}$ & $\begin{array}{l}\text { Drei Lerner/innen: Standbildbauer/in, } \\
\text { Mutter, } 2 \text { Stasi-Mitarbeiter/Polizisten }\end{array}$ \\
\hline
\end{tabular}

a Das ist nur ein Minimum. Die jeweiligen Gruppen können natürlich aus mehr Spieler/innen bestehen und somit gibt es auch mehr Interpretationsmöglichkeiten.

Jede Gruppe baut ihre Standbilder nun nacheinander auf. Die anderen beobachten. Die Lehrperson weist nochmals auf die Regeln hin, dass nicht gesprochen, die Mitspieler/innen geformt und die Mimik imitiert wird. Danach 
werden die Standbilder interpretiert. Die Deutung von Standbildern kann auf ganz verschiedene Weise geschehen. Hier ist eine Auswahl an Möglichkeiten: ${ }^{5}$

- Der/die Standbildbauer/in erläutert/deutet das Standbild.

- Das Publikum erläutert/deutet das Standbild. (Dieser Schritt kann vor oder nach der erst genannten Möglichkeit durchgeführt werden.)

- Der/die Standbildbauer/in oder ein/e Zuschauer/in nimmt die Perspektive einzelner Figuren im Standbild ein, indem er/sie deren Gefühle in dieser bestimmten Situation laut ausspricht ('Hilfs-Ich').

- Die Mitspieler/innen im Standbild interpretieren ihre Haltung, Mimik, Gestik, d.h., sie sagen aus ihren Rollen heraus, was sie gerade denken und empfinden.

- Das Publikum stellt den Mitspieler/innen Fragen.

- Die Lehrperson führt ein (provozierendes) Rollengespräch mit einer Figur im Standbild.

- Die Zuschauer/innen ahmen die Körperhaltung, Gestik und Mimik der Figuren im Standbild nach, um ein (körperliches) Gefühl für die Empfindungen und Gedanken der Figuren zu bekommen. Diese Vermutungen werden dann auf ein Signal hin laut ausgesprochen.

- Ein Text (z.B. ein Gedicht, ein Brief) wird von einer Person in der Standbildgruppe 'atmosphärisch' vorgelesen. An einer vorher festgelegten Textstelle kommen die restlichen Mitspieler/innen zum Standbild zusammen. Durch diese Kombination von Text und Bild entsteht eine starke Aussagekraft und Wirkung.

- Das Standbild wird zum Leben erweckt, die Figuren setzen die unterbrochene Handlung fort.

Szenen und Standbilder können auch mit Musik unterlegt werden. Die Auswahl der Musik hat grundsätzlich eine sehr starke/emotionale Wirkung und beeinflusst eine Spielszene, Handlung und Interpretation auf unterschiedliche Weise. Der Bau des Standbildes Nummer 5 (Tödliche Nachricht) kann beispielsweise mit Musik untermalt werden. Auch während der Interpretation dieses Standbildes, z.B. durch die Fortsetzung der Handlung in einer gespielten Szene, läuft die Musik. ${ }^{6}$

Ein weiterer Arbeitsauftrag könnte lauten, die Standbilder mit einem sorgfältig gewählten Titel zu präsentieren, um die Interpretation des Standbildes anzudeuten und auf den Arbeitsschritt 'Titelsuche- und diskussion' vorzubereiten.

\footnotetext{
${ }^{5}$ Das ist nur eine kleine Auswahl an Deutungsmöglichkeiten. In Scheller 1998: 59-68 und Schewe 2000: 87-89 gibt es ausführliche Beschreibungen für das Arbeiten mit Standbildern.

6 Das Lied The Host of Seraphim von der Band Dead Can Dance eignet sich sehr gut für diese Szene. Das Lied findet man auf dem Album A Passage in Time (2000) oder auf der 2003 veröffentlichten Best-of CD Wake von Dead Can Dance.
} 


\subsection{Schießbefehl: Titelsuche und -diskussion (Zeitdauer insgesamt ca. 50 Minuten plus individueller Zeitaufwand für die Hausaufgabe)}

Titelsuche (ca. 10 Minuten) - Der Text wurde den Lerner/innen in einem vorherigen Schritt ohne Titel gegeben, um verschiedene Interpretationsmöglichkeiten zu schaffen und die Vorstellungs- und Empfindungskraft der Lerner/innen im Prozess des Standbildbauens nicht einzuschränken. In diesem Schritt soll nun eine Diskussion zur Findung des Titels der Geschichte angeregt werden. Die Lehrperson kann zuerst fragen, ob es Vorschläge gibt, und diese dann im Plenum besprechen. Man kann auch verschiedene Titel vorgeben und fragen, welcher am besten auf die Kurzgeschichte passt und warum, z.B.: Der Fluchtversuch, Allein gelassen, Schießbefehl, Mutter und Sohn, Traum der Freiheit.

Dann wird mit den Lerner/innen der Titel Schießbefehl besprochen. Die DDR-Führung hat immer behauptet, dass es keinen Schießbefehl gegen MauerFlüchtlinge gegeben habe, obwohl das Gegenteil der Fall und dieser Umstand auch in der DDR lange bekannt war. Nach dem Ende der DDR wurde das offiziell bestätigt. Sowohl 1993 als auch 2007 wurde in Stasi-Unterlagen ein bedingungsloser Schießbefehl entdeckt: „Zögern Sie nicht, auch nicht bei Frauen und Kindern. “"

Erweiterungsmöglichkeit: Internetrecherche (Einführung und Auswertung ca. $40 \mathrm{Mi}$ nuten) - Auf der Website Chronik der Mauer (http://www.chronik-dermauer.de/) von der Bundeszentrale für politische Bildung gibt es unter dem Link 'Lernen' die 'Guided Tour' unter anderem zum Thema 'Schießbefehl' (Thema 14 auf der Webseite). Eine Erweiterungsmöglichkeit hier wäre eine Internetrecherche, die als Hausaufgabe durchgeführt wird. Unter dem Link 'Schießbefehl' findet man ein Textdokument mit dem Titel: Über den DDRSchießbefehl, der nie existierte (von Hans-Hermann Hertle). Da der Text lang ist, könnte man ihn in Abschnitte aufteilen und die Fragen dazu in Gruppen beantworten lassen. Im Plenum werden dann alle Informationen ausgetauscht. Die Einführung in den Text von Hertle und die zu bearbeitenden Themen/Fragen sollten im Unterricht besprochen werden. In Gruppen werden die Fragen als Hausaufgabe bearbeitet und dann gemeinsam besprochen und reflektiert. Die Lerner/innen können z. folgende Fragen beantworten:

- Wer war Erich Honecker? Was war seine Funktion im DDR-Staat?

- Wie viele Menschen sind an der deutsch-deutschen Grenze und an der Berliner Mauer zwischen 1949 und 1989 umgekommen?

- Wie viele Menschen wurden bei einem Fluchtversuch erschossen?

- Durch welche anderen Gewalttaten und Umstände sind DDR Flüchtlinge ums Leben gekommen?

\footnotetext{
7 Das Zusatzmaterial im Anhang D und E - ein Zeitungsartikel aus dem Fokus und eine thematische Einführung von Cornelsen - sind als Hintergrundinformationen für die Lehrperson gedacht.
} 
- Wie hieß der Befehl, den DDR-Grenzsoldaten täglich erhielten?

- Was passierte, wenn ein DDR-Grenzsoldat einen Flüchtling erschoss?

- Wie hieß das letzte Maueropfer der DDR und wann wurde er/sie erschossen?

- Wer war Erich Mielke? Was war seine Funktion im DDR Staat?

- Ende April 1989 äußerte sich Erich Mielke folgendermaßen: „Wenn man schon schießt, dann muß man das so machen, daß nicht der Betreffende noch wegkommt, sondern dann muß er eben dableiben bei uns. (...)". Was meint Mielke damit? Welche Haltung drückt er mit dieser Äußerung aus?

- Der Titel dieses Textes heißt: Über den DDR-Schießbefehl, der nie existierte. Was bedeutet das?

\subsection{Einfühlung in eine Rolle: Rollenbiographie und Heißer Stuhl (Zeitdauer insgesamt ca. 45 Minuten plus individueller Zeitaufwand für die Hausaufgabe)}

Eine Rollenbiographie schreiben (Einführung und Besprechung des Fragenkatalogs ca. 15 Minuten) - Die Einfühlung in und Identifikation mit einer Rolle können durch Standbilder und deren Interpretation, durch das Verfassen einer Rollenbiographie oder die Methode des Heißen Stuhls erfolgen (cf. Strasberg 1988). Um eine erfolgreiche Identifikation eines/r Schauspielers/in mit seiner oder ihrer Rolle zu erreichen, kann z.B. mit den folgenden vier Fragen gearbeitet werden:

- Wer ist die Figur/Person?

- Wo ist die Figur/Person?

- Was tut die Figur/Person dort?

- Was ist geschehen, bevor die Figur/Person hierher kam (Umstände)?

Damit sich die Lerner/innen noch besser an die Personen und Geschehnisse in der Geschichte Schießbefehl annähern und sich in sie 'einfühlen' können, bietet es sich also an, diese Fragen zu beantworten. Dazu schreiben die Lerner/innen eine Rollenbiographie für eine der Personen im Text. Die Rollenbiographie dient zugleich als Vorbereitung auf die letzte szenische Improvisation mittels der Technik des Heißen Stuhls (s.u.). In ihrer Rollenbiographie sollen die Lerner/innen beschreiben, wie die Person ihrer Wahl lebt, wo sie lebt, wie alt sie ist, wie ihre Sozialisation verlaufen ist, welche Beziehungen sie unterhält etc. 
Für diese Aufgabe orientieren sich die Lerner/innen zuerst an der literarischen Vorlage. Wir wissen z.B., dass der Sohn im Text Schießbefehl Gerhard heißt, dass er ca. 19 oder 20 Jahre alt ist (er hat gerade das Facharbeiterabitur gemacht und sollte zur Armee gehen), dass er ein Motorrad hat und sich (vielleicht) erhängt hat. Wir wissen aber nicht, wie er lebte, wie sein Zimmer aussah, wer seine Freunde waren, wie sein Alltag aussah, welche Musik er gern hörte oder wie er aufgewachsen ist. Diese Leerstellen des Textes werden mithilfe von Fragen zur Einfühlung in die Figur geschlossen. ${ }^{8}$

Zusätzlich verarbeiten die Lerner/innen in ihren Rollenbiographien auch Material, das sie durch andere Texte, Filme, Bilder oder Erzählungen über das Leben in der DDR erfahren haben Die Rollenbiographie wird aus der Ich-Perspektive geschrieben und in kompletten Sätzen: „Das fördert die Identifikation, hilft eigene Erlebnisse, Fantasien, Empfindungen, Denk- und Schreibmuster zu aktivieren und mit zu verarbeiten" (Scheller 2004: 62). Die folgende Auflistung, die beliebig erweitert, modifiziert oder reduziert werden kann (und sollte), hilft, den Schreibprozess zu steuern. Schewe (1998: 51) weist darauf hin, dass es keinen festen Fragenkatalog gibt, der auf jede Figur anwendbar ist. Vielmehr müsse man die Einfühlungsfragen auf die jeweiligen Figuren eines bestimmten Textes oder einer Situation zuschneiden. Bei den folgenden Fragen nähern sich die Lerner/innen ihrer Person zuerst von außen. Sie konzentrieren sich auf die äußeren Rahmenbedingungen wie Name, Alter, Familiengefüge der Figur, bevor man deren innere Haltungen und Einstellungen (Wünsche, Ängste, Gefühle) verortet. ${ }^{9}$ In Schießbefehl sind die Gefühle und Gedanken der Personen nicht beschrieben außer, dass man am Anfang erfährt, dass die Mutter sich Sorgen macht, weil Gerhard nicht zurückgekommen ist. Die Lerner/innen müssen also aus der Situationsbeschreibung und mithilfe der zuvor inszenierten Standbilder sowie ihrer eigenen Erfahrungen und Erlebnisse Rückschlüsse auf die emotionalen Befindlichkeiten der Charaktere ziehen.

\section{Äußere Welt der Figur}

- Name, Alter, Wohnung: Wie heißt du? Wie alt bist du? Wo, mit wem und wie wohnst du (Größe, Zimmeranzahl, Einrichtung, Möbel, Bilder, Pflanzen, Haustiere)?

- Sozialisation: Hast du Eltern und Geschwister? Wo und wie hast du in deiner Kindheit/Jugend gewohnt? Wie war dein Vater/deine Mutter? Waren sie Vorbilder für dich? Habt ihr viel gemeinsam unternommen?

- Familie: Bist du verheiratet? Hast du Kinder? Was bedeutet dir deine Familie? Wie viel Zeit verbringt ihr miteinander und was macht ihr dann?

- Beruf und Geld: Was ist dein Beruf? Wo arbeitest du und wie lange arbeitest du dort schon? Wie sieht dein (Berufs)Alltag aus? Welche

\footnotetext{
${ }^{8}$ Zur Bedeutung der Leerstellen bei der Sinnkonstruktion während des Lesens cf. z.B. Lensch 2000 sowie Richter 1996: 517.

${ }^{9}$ Für weitere Einfühlungsfragen cf. Scheller 1998: 174-177 zbd 2004: 62-66.
} 
Tätigkeiten führst du aus und welche Verantwortung hast du? Wie sind deine Kollegen - versteht ihr euch? Übst du deinen Beruf gerne aus? Wie ist deine materielle Situation?

- Freizeit und Freunde: Hast Du Hobbys? Was machst du in deiner Freizeit? Reist du viel? Hast du Freunde? Triffst du dich mit ihnen (wann, wo, wie oft, was macht ihr dann)? Was ist dir in einer Freundschaft wichtig?

- Politik: Bist du politisch (welche Themen interessieren dich)? Bist du in der Partei/SED? Sind die gegenwärtigen politischen Verhältnisse (in der DDR) okay für dich? Was hältst du von der Mauer, die Ost und West voneinander abgrenzt?

\section{Innere Welt der Figur}

- Wünsche, Ängste und Fantasien: Welche Wünsche und Fantasien hast du? Träumst du (am Tag, in der Nacht)? Wenn du an einem Ort deiner Fantasie sein könntest, wo wäre das, wie sieht es dort aus, wer ist da mit dir? Hast du manchmal Angst? Wovor? Bis du manchmal wütend und aggressiv? Wie gehst du mit deinen Ängsten und Aggressionen um?

- Momentane Befindlichkeiten, Intentionen und Gedanken:Was machst du gerade (jetzt in diesem Moment)? Was geht dir dabei durch den Kopf? Welche Empfindungen bewegen dich dabei/was fühlst du? Fühlst du dich hier (wo du gerade im Moment bist) wohl/unwohl? Was beschäftigt dich jetzt gerade am meisten? An wen denkst du gerade? Was hast du vor?

Diese Fragen helfen, den Schreibprozess zu steuern und die Aufmerksamkeit auf Lebensbereiche, soziale Umfelder und innere Haltungen zu richten, die für das Verständnis der verschiedenen Figuren im Text wichtig sind. Während des Unterrichts gibt es leider meist nicht genügend Zeit, um sich eingehender damit zu beschäftigen, deshalb eignet sich das Schreiben einer Rollenbiographie gut als Hausaufgabe. Daran kann sich in einem letzten Schritt die szenische Methode Heißer Stuhl anschließen. Die Lerner/innen gaben beim Feedback an, dass die Rollenbiographie und der Heiße Stuhl für sie am effektivsten waren. ${ }^{10}$

Der Heiße Stuhl (ca. 20-30 Minuten) — Durch die Technik des Heißen Stuhls können nun die Vorstellung und Einfühlung in eine Figur öffentlich gemacht werden. Die Lerner/innen in ihrer Rolle als Mutter, Vater, Gerhard oder Polizist begeben sich auf den Heißen Stuhl (ein Stuhl, der in die Mitte oder vor einen Halbkreis gestellt wird). Eine Erweiterung besteht darin, die Lerner/innen

\footnotetext{
10 Cf. Anhang F für Umfrageergebnisse eines Feedbackfragebogens, den ich nach Beendigung der Unterrichtseinheit an die Lerner/innen verschickt habe. Dort sind ebenfalls drei Beispielrollenbiographien einzusehen: aus der Sicht von Gerhard, aus der Sicht eines Nachbarn und aus der Sicht eines Grenzsoldaten. Ich habe die Rollenbiographien so übernommen, wie sie mir die Lerner/innen geschickt haben, d.h. mit kleinen Fehlern.
} 
zu bitten, schon auf dem Weg zum Heißen Stuhl in ihrer Rolle zu agieren. Bestimmte Rahmenbedingungen, soziale Rollen und Gefühle schlagen sich in Körper- und Gehhaltungen nieder. Beim bewussten Gang zum Stuhl, z.B. in der Rolle der Mutter (angespannt, traurig, wütend, steif, unter Schock etc.) oder in der des Stasimitarbeiters (aufrecht, maskulin, sich seiner Sache sicher und seiner Verantwortung dem SED Staat gegenüber bewusst), werden über die Körpererinnerung Empfindungen aktiviert, die wiederum helfen, sich in eine bestimmte Person einzufühlen.

Der Person auf dem Heißen Stuhl werden nun durch das Publikum Fragen gestellt, z.B. zur Person (Alter, Sozialisation, Freunde etc.; s. Fragenkatalog und Rollenbiographie), zur Situation (Leben in der DDR, Fluchtversuch, Grenze) und zu den Gedanken, Handlungen und Empfindungen der jeweiligen Personen in der Geschichte („Mensch Gerhard, Du solltest doch zur Armee gehen am Montag, warum biste denn jetzt abgehauen?“ oder „Dein Sohn Gerhard ist tot. Glaubst Du die Version, die Dir die Stasi über seinen Tod erzählt hat?" oder „Du bist Grenzsoldat in der DDR. Was hast Du denn gedacht, als Du Gerhard beim Fluchtversuch geschnappt hast?"). Die Funktionen des Heißen Stuhls sind: a) sich in der Rolle nochmals ganz klar zu werden über seine Figur und b) verschiedene Situationen/Szenen für die Person auf dem heißen Stuhl und das Publikum verständlich und transparent zu machen (hier kommen die verschiedenen Interpretationsmöglichkeiten ins Spiel: von dem Szenario, wie es tatsächlich in Schießbefehl beschrieben ist, über die Version, dass Gerhard gar nicht bis ins Gefängnis gekommen ist, weil er schon an der Grenze erschossen wurde, bis zur Möglichkeit, dass er es über die Grenze geschafft hat, jetzt im Westen lebt, aber jeglicher Kontakt zur Mutter unmöglich ist und die Stasi die Mutter belügt, wenn sie sagt, dass ihr Sohn tot sei.). Weiterhin gibt der Heiße Stuhl den Fragesteller/innen im Publikum die Möglichkeit, ihre Meinung zu einer Figur zu äußern („Glaubst Du wirklich, du dienst deinem Staat, indem du Menschen abknallst?"). Die Fragevorschläge verdeutlichen, dass die Grundvoraussetzung für diese Methode ein gutes und offenes Gruppenklima ist. Herrscht beispielsweise eine wenig vertraute und vertrauensvolle oder gar aggressive Atmosphäre, kommt der Heiße Stuhl eher einem Kreuzverhör gleich.

\section{Reflexion}

Feedbackrunden und Reflexionen dienen dazu, Reflexionskompetenz zu erwerben und konstruktive Kritikfähigkeit zu schulen. Sie sind wichtiger Teil des Lernprozesses: Die gemachten Erfahrungen zu reflektieren, bedeutet, diese zu ordnen, zu vertiefen und zu speichern. Somit werden Erfahrungen vergleichbar und für zukünftiges Erleben abrufbar. Im Fremdsprachenunterricht wird durch regelmäßiges Feedback außerdem der Wortschatz der Lerner/innen erheblich erweitert und gefestigt. In meiner Unterrichtspraxis haben sich das Stundenprotokoll und das Lerntagebuch als Reflexionsmethoden bewährt. Sie helfen, das Erlebte zu dokumentieren und zu verarbeiten. Das Stundenprotokoll wird nach jeder Unterrichtsstunde auf Deutsch skizziert. Es beinhaltet zwei Teile: 
Rekonstruktion und Reflexion. Die Lerner/innen notieren sich stichwortartig, was sie gemacht haben, welche Methoden benutzt wurden, welcher Text interpretiert wurde etc. und wie sie sich dabei gefühlt haben. Diese Skizzen dienen dann als Vorlage und Erinnerung für den Eintrag ins Lerntagebuch. Das Lerntagebuch orientiert sich an denselben Fragen wie das Stundenprotokoll, ist aber im Gegensatz dazu als vollständiger Text zu formulieren. Der vollständige Eintrag ins Lerntagebuch findet nach jeder abgeschlossenen Einheit statt.

Die folgenden Punkte dienen zur Orientierung, um die Erfahrungen im Unterricht möglichst systematisch im Stundenprotokoll und später im Lerntagebuch zu verarbeiten:

\section{Teil I: Textarbeit}

- Eine kurze Zusammenfassung/Rekonstruktion des Textes oder Themas, den/das wir gelesen und inszeniert haben.

- Reflexion des Textes: Hat der Text mich angesprochen? Warum? Warum nicht? Gibt es einen Charakter im Text, der mich besonders interessiert? Warum? War der Text leicht, mittel oder schwierig? Welche neuen Wörter und Redewendungen habe ich gelernt (Beispiele)?

\section{Teil II: Reflexion der Unterrichtsmethode und des eigenen Erfahrungspro- zesses}

- Eine kurze Rekonstruktion der Unterrichtsstunden: Was haben wir gemacht? Wie haben wir es gemacht? Wie war die Stimmung in der Klasse? Wie ging es mir dabei?

- Reflexion der Methoden: Haben die ausgewählten Methoden und Elemente (z.B. Standbilder oder Rollenbiographie) mir den Zugang zum Text erleichtert? Welche Methoden finde ich gut und warum, welche nicht?

- Reflexion des eigenen Lernens: Was für ein Lernertyp bin ich? Lerne ich besser, gleich gut oder schlechter mit allen Sinnen? Welche Intelligenzen wurden (primär) angesprochen? Was habe ich konkret gelernt? Welchen Ertrag hat die Einheit für meinen Sprachlernprozess?

Da die Deutschkurse am Goethe-Institut keine 'Notenkurse' sind, d.h., da es keine Benotung für (Haus)Aufgaben, Projekte etc. gibt, werden auch das Stundenprotokoll und das Lerntagebuch nicht bewertet. Ich biete meinen Lerner/innen an, ihre Aufzeichnungen durchzusehen, und mache Vorschläge für sprachliche Verbesserungen. Das heißt aber auch, dass nicht alle Lerner/innen das Stundenprotokoll bzw. Lerntagebuch konsequent führen. ${ }^{11}$

\footnotetext{
${ }^{11}$ In Retzlaff (2008) habe ich eine dramapädagogische Unterrichtseinheit mit Student/innen an der Universität beschrieben, deren Leistungen im Deutschunterricht bewertet wurden. Dort findet man im Anhang die Bewertungskriterien und Anforderungen für diesen 'credit course'.
} 


\title{
5 Feedback
}

Die Unterrichtseinheit zum Thema 'Mauer' wurde mit Hilfe eines halbstrukturierten Fragebogens evaluiert. Dazu wurde der Fragebogen an alle Lerner/innen aus meinen Deutschkursen im Goethe-Institut verschickt (B1 Kurs: 8 Lerner/innen; C1 Kurs: 6 Lerner/innen) sowie an die Lehrer/innen der Fortbildungen (ca. 25 Teilnehmer/innen). ${ }^{12}$ Er ist auf Englisch verfasst, da ich diese Einheit auf Deutsch und Englisch unterrichtet habe. Da der Rücklauf mit nur 15 Bögen nicht besonders hoch war, sind die Zahlen (s. Anhang F, Tabelle) nicht repräsentativ, aber zeigen zumindest eine Tendenz auf: Der dramapädagogische Ansatz hilft offensichtlich vielen Lerner/innen, sich auf eine emotionale Ebene zu begeben und mit allen Sinnen zu lernen. Es ist interessant, dass die Aktivitäten Lückentext, Rollenbiographie und Heißer Stuhl als ganz besonders effektiv bewertet wurden. Es ist zu vermuten, dass sie beliebter sind als z.B. Standbilder oder die Improvisation zur Touristenführung, weil sie weniger spontanes Sprechen und Improvisieren erfordern, sondern hier in überschaubaren Strukturen (z.B. Fragenkatalog) gearbeitet wird und eine Vorbereitungszeit vorgesehen ist.

\section{Fazit}

Dramapädagogik und szenische Improvisation sind kreative Lern- und Lehrmethoden, die eine Alternative zum traditionellen Frontalunterricht darstellen und Offenheit für kooperative und 'sinnesreiche' Unterrichtsformen von allen Beteiligten einfordern. Es war mein Ziel, die Lerner/innen emotional und auf vielen Sinneskanälen an das Thema 'DDR und Leben mit der Mauer' heranzuführen. Dies wurde größtenteils durch die Anwendung einer Vielzahl von dramapädagogischen Übungen erreicht, wie die Aussage einer Teilnehmerin deutlich macht:

\begin{abstract}
The wall is something that always came up in regular conversations, as a form of government oppression and political violence; it came as a debate between socialism and capitalism and there was always a disconnect. This time however, I felt a certain emotional connection - I could not pretend that no this is just a charade; it almost felt real!
\end{abstract}

Einige Lerner/innen erwähnten aber auch, dass Dramapädagogik ein gewöhnungsbedürftiger Ansatz sei, da er emotional überwältigt (was sicher auch am Thema selbst lag). Ein einziger Teilnehmer (sehr gutes Sprachniveau C1) empfand diese Einheit als überflüssig und als „having only a small correlation to the language learning course". Im folgenden Feedback-Auszug dieses Lerners wird klar, dass der traditionelle Ansatz häufig fest verankert ist und als die bessere Methode angesehen wird, eine Fremdsprache zu lernen:

\footnotetext{
12 Der für das Feedback benutzte Fragebogen sowie einige ausgewählte Kommentare von Teilnehmer/innen befinden sich wie drei Rollenbiographien im Anhang (F) des Textes.
} 
For me personally, it would have been more helpful for my learning needs, if for example one could provide one written short scene out of Thomas Brussig's novel Am kürzeren Ende der Sonnenallee and ask the participants one or two questions from these proposed above or another question that could evoke a critical but balanced thinking. The participants would then need to reply in writing (say within 30-45 min) without a dictionary or any book. Then, one could orally discuss it and finally in the subsequent lesson receive corrections to the mistakes in the written texts.

Der Schwerpunkt der Dramapädagogik und szenischen Improvisation liegt vor allem im Prozess, im Entwickeln, Ausprobieren, Improvisieren, im Perspektivwechsel und im Einbringen eigener Erfahrungen und Gefühle. Dieser Ansatz erfordert von den Teilnehmenden eine ungewohnte Arbeitsweise zuzulassen. Von der Lehrperson verlangt es Selbstbewusstsein, Risikobereitschaft und gruppendynamische Sensibilität.

Das Allgemeinziel, die Sprachkompetenz der Lerner/innen zu schulen und zu verbessern, wurde hauptsächlich durch das Schreiben und Verbalisieren der Rollenbiographie erreicht. Auch Lernende mit weniger guten Deutschkenntnissen konnten innerhalb ihrer Rolle agieren und reagieren. Beim Einsatz von Improvisationen (z.B. bei den Sprechhaltungen), bei denen es darum ging, in einer ersten Auseinandersetzung mit dem Thema und den Figuren aus Schießbefehl ungewöhnliche Haltungen und fremde Handlungsmuster zu entwickeln und zu erproben, kamen bei einigen Lerner/innen Blockaden auf. Einige haben sich schlicht nicht getraut, anderen fehlten die sprachlichen Strukturen oder, wie sie glaubten, der Wortschatz. Mein Eindruck ist eher, es fehlte an einer Vertrautheit mit diesem, für die meisten Lerner/innen ungewohnten Arbeiten im Fremdsprachenunterricht. Dramapädagogik basiert auf der Vorstellung, dass „natürliche Interaktionssituationen beim Handeln in fiktiven Kontexten entstehen" (Schewe 1993: 210). Der Einsatz von Techniken aus dem szenischen Spiel und der Dramapädagogik schafft diese lebensnahen Situationen und befördert das Sprachenlernen: „Fiktive Welten [sind] sprachfördernd, denn es entstehen Situationen, auf die emotional und flexibel reagiert werden muss" (Vaninetti 2005: 5).

\section{Literatur -}

Gandhi, Mahatma (1958): All Men Are Brothers. Life and Thoughts of Mahatma Gandhi as Told in His Own Words. UNESCO Paris.

Hertle, Hans-Hermann (1999): Über den DDR Schießbefehl, der nie existierte. http://www.chronik-der-mauer.de/index.php/de/Media/ TextPopup/id/856754/oldAction/Index/oldId/783138/oldModule/ Start/page/ 0 (27. November 2009; Erstveröffentlichung: Berliner Morgenpost, 28. März 1999).

Lensch, Martin (2000): Spielen, was (nicht) im Buche steht. Die Bedeutung der Leerstelle für das literarische Rollenspiel. Münster: Waxmann. 
Mettenberger, Wolfgang (2004): Tatort Theater. Weinheim: Deutscher Theaterverlag.

Retzlaff, Steffi (2008): Fitness for the Muscles and the Mind: Incorporating Nia (Neuromuscular Integrative Action) into a Drama-Pedagogical Teaching Unit on Thomas Brussig's Novel Am kürzeren Ende der Sonnenallee. In Scenario 1/2008. http://publish.ucc.ie/scenario/2008/ 01/retzlaff/04/en.

Richter, Matthias (1996): Wirkungsästhetik. In: Arnold, Heinz Ludwig; Detering, Heinrich (Hrsg.): Grundzüge der Literaturwissenschaft. München: Dt. Taschenbuch-Verl., 516-535.

Scheller, Ingo (2004): Szenische Interpretation. Selze-Velber: Kallmeyer-

Scheller, Ingo (1999): Erfahrungen (re)inszenieren: Szenische Interpretation von Texten in der schulischen und außerschulischen Bildungsarbeit. In: Lecke, Bodo (Hrsg.): Literatur und Medien in Studium und Deutschunterricht. Frankfurt a.M.: Europäischer Verlag der Wissenschaften, 319-339.

Scheller, Ingo (1998): Szenisches Spiel. Berlin: Cornelsen.

Scheller, Ingo (1989): Wir machen unsere Inszenierungen selber I. Oldenburg: Zentrum für pädagogische Berufspraxis.

Schewe, Manfred L. (2000): DAF-Stunden dramapädagogisch gestalten Wie mache ich das? In: Schlemminger, Gerald; Brysch, Thomas; Schewe, Manfred (Hrsg): Pädagogische Konzepte für einen ganzheitlichen DaF-Unterricht. Berlin: Cornelsen, 72-105.

Schewe, Manfred L. (1998): Serie Fremdsprache inszenieren (1-2). Teil 1: Einfühlungsfragen beantworten; Teil 2: Rollenmonolog. In: Fremdsprachenunterricht 42/51, 51-52.

Schewe, Manfred L. (1993): Fremdsprache inszenieren. Zur Fundierung einer dramapädagogischen Lehr- und Lernpraxis. Oldenburg: Carl von Ossietzky-Universität.

Strasberg, Lee (1988): Ein Traum der Leidenschaft. Die Entwicklung der "Methode". München: Schirmer-Mosel.

Vaninetti, Sara (2005): Fremdsprache im Spiel. Für einen dramapädagogischen Fremdsprachenunterricht. http://www.theaterwerkstatt-heidelberg.de/uploadverzeichnis 
Weblinks -

www. mauerfall-berlin.de

wWw. chronik-der-mauer.de/

http://www.berlinermaueronline.de/geschichte/mauerfall.htm

„Eingemauert“ Empfehlenswertes animiertes Video (10 Minuten) von der Deutschen Welle über die innerdeutsche Grenze: http://www youtube. $\mathrm{com} /$ watch?v=jlbAUFvh04k

Goethe-Institut Kanada: http://www.goethe.de/ins/ca/lp/prj/grh/wdm/ deindex.htm

Goethe-Institut Deutschland: http://www.goethe.de/lhr/prj/kal/bem/ deindex.htm 


\section{Anhang A: Schießbefehl}

Ich fahre zum Vater, sagte er, nimmt das Motorrad, und ich denke, warum kommt er denn nicht wieder, wo der bloß bleibt, langsam werde ich unruhig, da kommen die und sagen, ich soll nach P....kommen, er hat über die Grenze gewollt, und sie haben ihn erwischt.

Also bin ich mit dem nächsten Zug nach P....gefahren, er hat schon gestanden, sagen sie, und als ich mich nicht mehr beherrschen konnte und mir die Tränen kamen, haben sie gesagt, machen Sie sich keine Sorgen, gute Frau, Ihr Gerhard lebt, er hat gut gegessen, und jetzt schläft er.

Und wenn's während der Armeezeit gewesen wäre, wär's schlimmer.

Er hatte doch gerade erst seinen Facharbeiter mit Abitur gemacht, und am Montag sollte er einrücken....

Und dann, am Montagnachmittag, kommen die von hier und sagen, ich soll am Dienstag nach P....kommen.

Ich backe einen Kuchen, kaufe ein, und dann sagen sie mir in P..., ob ich denn nichts wüßte, ob denn unsere nichts gesagt hätten, er hat sich erhängt.

Mit der Unterhose.

Und sie hätten ihm einen Zettel gegeben, ob er mir nicht ein paar Worte schreiben wollte, aber er hätte abgelehnt.

Wie er mir das hat antun können....

Und sehen darf ich ihn nicht, nur noch kurz vor der Feier, die im Gefängnis stattfindet.

Aushändigen können sie mir nur die Urne.

Reiner Kunze: Die wunderbaren Jahre (Fischer Verlag).

\section{Anhang B: Textschnipsel}

- Ich fahre zum Vater, sagte er

- ich denke, warum kommt er denn nicht wieder

- da kommen die und sagen, er hat über die Grenze gewollt

- sie haben ihn erwischt

- machen Sie sich keine Sorgen, gute Frau, Ihr Gerhard lebt

- am Montag sollte er einrücken

- ich backe einen Kuchen

- dann sagen sie mir in P..., ob ich denn nichts wüßte 
- er hat sich erhängt

- Wie er mir das hat antun können....

- Und sehen darf ich ihn nicht

- Aushändigen können sie mir nur die Urne

\section{Anhang C: Lückentext}

Wer sagt oder tut was? Bitte ordnen Sie den Personalpronomen die jeweilige Person zu: Mutter, Sohn Gerhard, Polizei „von hier“ oder Polizei/Stasi in P...

Ich [__ _ ] fahre zum Vater, sagte er [___ ], nimmt das Motorrad, und ich [__ ] denke, warum kommt er [__ $]$ _ denn nicht wieder, wo der [ _ _ ] bloß bleibt, langsam werde ich [__ ] unruhig, da kommen die [__ ] und sagen, ich [__ ] soll nach P....kommen, er [ _ _ ] hat über die Grenze gewollt, und sie [__ ] haben ihn [ _ _ ] erwischt. Also bin ich [__ ] mit dem nächsten Zug nach P....gefahren, er [ __ _ ] hat schon gestanden, sagen sie [___ ], und als ich [ _ ] mich nicht mehr beherrschen konnte und mir die Tränen kamen, haben sie [___ ] gesagt, machen Sie sich keine Sorgen, gute Frau, Ihr Gerhard lebt, er hat gut gegessen, und jetzt schläft er. Und wenn's während der Armeezeit gewesen wäre, wär's schlimmer.

Er [ _ $]$ hatte doch gerade erst seinen Facharbeiter mit Abitur gemacht, und am Montag sollte er [ _ _ ] einrücken....Und dann, am Montagnachmittag, kommen die von hier [__ _ ] und sagen, ich [

] soll am Dienstag nach P....kommen. Ich [____ ] backe einen Kuchen, kaufe ein, und dann sagen sie [__ $]$ mir in P..., ob ich denn nichts wüßte, ob denn unsere [__ ] nichts gesagt hätten, er [__ _ ] hat sich erhängt. Mit der Unterhose. Und sie [ einen Zettel gegeben, ob er [ schreiben wollte, aber er [ ] mir [ _ _ ] nicht ein paar Worte können.....Und sehen darf ich [__ _ ] ihn [__ ] ] nicht, nur noch kurz vor der Feier, die im Gefängnis stattfindet. Aushändigen können sie [ ] mir nur die Urne.

\section{Anhang D: Schießbefehl ohne Rücksicht auf Kinder}

Ein jetzt aufgetauchtes Dokument belegt, wie radikal die DDR einst ihre Grenze sicherte. Einsatzkommandos sollten auch auf fliehende Frauen und Kinder schießen.

Die Magdeburger Außenstelle der Birthler-Behörde entdeckte 46 Jahre nach Beginn des Mauerbaus offenbar ein brisantes Dokument. Die „Magdeburger Volksstimme" vom Samstag veröffentlichte Auszüge aus einer siebenseitigen Dienstanweisung vom 1. Oktober 1973. Das Dokument belegt, wie rücksichtslos 
einer 1968 gebildeten „Einsatzkompanie“ des Ministeriums für Staatssicherheit das Schießen befohlen worden sei. Die Stasi-Kompanie wurde auf Befehl des Leiters der Hauptabteilung I im Ministerium für Staatssicherheit 1968 gebildet und bestand bis 1985. Ihre Mitglieder sind dem Bericht zufolge speziell ausgebildete Einzelkämpfer gewesen, die in die Grenztruppen eingeschleust wurden und dort Grenzdurchbrüche verhindern sollten.

IM in Uniform als brutale Grenzwächter

In dem Papier heißt es demnach wörtlich: „Zögern Sie nicht mit der Anwendung der Schusswaffe, auch dann nicht, wenn die Grenzdurchbrüche mit Frauen und Kindern erfolgen, was sich die Verräter schon oft zu nutze gemacht haben." Der bis 1985 bestehenden Einsatzkompanie hätten zuletzt rund 60 hauptamtliche Inoffizielle Mitarbeiter im besonderen Einsatz (HIME) angehört, die als normale Soldaten getarnt, aber von der Stasi bezahlt worden seien, hieß es weiter.

Der Außenstellen-Leiter Jörg Stoye spricht von einem Aufsehen erregenden und höchst bedeutsamen Fund. Bisher habe es in den offiziellen DDRAkten einen so deutlichen Schießbefehl, der keinerlei Einschränkung des Schusswaffengebrauchs vorsehe, nicht gegeben.

Quelle: Fokus online 11.08.2007 http://www.focus.de/politik/deutschland/ddrgrenze_aid_69505.html.

\section{Anhang E: Die Debatte um DDR-Schießbefehl für Stasi-Grenzer}

Zum Dokumentenfund - Bei dem der Öffentlichkeit am 11. August 2007 präsentierten Fund der Magdeburger Außenstelle der Stasi-Unterlagenbehörde handelt es sich um einen schriftlichen Auftrag, den ein Stasi-Unterfeldwebel am 1. Oktober 1973 seinem Vorgesetzten, einem Stasi-Oberstleutnant, quittierte. Nach diesem Auftrag sollte der Unterfeldwebel, der als Hauptamtlicher Inoffizieller Mitarbeiter (HIM) der MfS-Spezialeinheit „Einsatzkompanie“ in ein DDR-Grenzregiment eingeschleust werden sollte, Fluchtversuche anderer Grenzsoldaten verhindern. Die Leiterin der Birthler-Behörde räumte jetzt ein, dass das Dokument ihrem Haus bereits anderthalb Jahre bekannt war, ehe es am 11. Juni 2007 der „Magdeburger Volksstimme“ zur Verfügung gestellt worden war. Die Regionalzeitung veröffentlichte es dann zwei Monate später, unmittelbar vor dem Jahrestag des Mauerbaus 2007.

Der Einsatzauftrag an den Unterfeldwebel war zwar bislang nicht bekannt, der Inhalt des Dokuments ist jedoch nicht neu. Ein wortgleicher Befehl an einen anderen verdeckten Stasi-Einzelkämpfer der Sondereinheit „Einsatzkompanie“ aus dem Jahre 1974 ist bereits vor zehn Jahren in einem Dokumentenband zur DDR-Geschichte publiziert worden (cf. M 1). Marianne Birthler übernahm nun die Verantwortung dafür, dass der Magdeburger Fund, von ihr fälschlich als „neu“ bezeichnet, bis zu seiner öffentlichen Freigabe weder in Magdeburg noch in Berlin mit dem wissenschaftlichen Kenntnisstand der Stasi-Behörde abgeglichen wurde. 
Zur MfS-Spezialeinheit „Einsatzkompanie“ - Die MfS-Angehörigen der Spezialeinheit „Einsatzkompanie“ waren der Stasi-Hauptabteilung I, Äußere Abwehr zugeordnet. Diese Spezialeinheit wurde 1968 gebildet und sollte, neben anderen Aufgaben wie der Verhinderung von Terror- und Gewaltverbrechen, die Fahnenflucht von Soldaten verhindern. Ihr gehörten anfangs ca. 30 Mitarbeiter an, später durchschnittlich 50-70, die zum Teil konspirativ in Truppenteile der NVA eingeschleust worden waren. Die Angehörigen der MfS-Spezialeinheit mussten, wenn sie getarnt in normale Grenzeinheiten geschleust wurden, Einsatzaufträge - wie der Magdeburger Fund belegt - unterzeichnen. Die Einheit wurde 1985 aufgelöst (cf. M 2).

Zu juristischen Konsequenzen - Der Magdeburger Staatsanwaltschaft war der Aktenfund vor seiner Veröffentlichung am 11. August 2007 nicht bekannt. Die Behörde prüft zurzeit die Zuständigkeit und erklärte, Ermittlungen seien grundsätzlich denkbar. Es ist allerdings davon auszugehen, dass der gefundene Einsatzauftrag vorerst in kein neues Ermittlungsverfahren münden wird, denn die noch vorhandenen Unterlagen der für gezielte Tötungen, Entführungen und andere illegale Operationen zuständigen MfS-Einheit „Einsatzkompanie“ sind bereits im Rahmen eines aufwändigen Ermittlungsverfahrens ausgewertet worden. Dabei war es konkret um den Mord an dem DDR-Flüchtling Michael Gartenschläger gegangen (cf. M 2). Da es sich bei den gefundenen Dokumenten um konkrete MfS-Einsatzaufträge handelt, die nur für einen kleinen Kreis ausgewählter Stasi-Einzelkämpfer galt und nicht für alle Grenzsoldaten, ist eine Neuauflage der Prozesse um die Todesschüsse an der innerdeutschen Grenze nicht zu erwarten (cf. M 3).

Inzwischen erklärte die Birthler-Behörde, dass ein Abgleich mit der Registratur des Hauses am 13. August 2007 ergab, dass ein inhaltsgleiches Dokument zum ersten Mal 1993 als „Mitteilung ohne Ersuchen“ an die Berliner Staatsanwaltschaft übergeben worden war. Nach dem Stasi-Unterlagen-Gesetz ist die Behörde verpflichtet, von sich aus die zuständigen Stellen zu informieren, wenn sich im Rahmen ihrer Recherchen Anhaltspunkte für eine Straftat im Zusammenhang mit der Tätigkeit des Staatssicherheitsdienstes ergeben. Auch dieses Dokument enthält die Unterschrift des Empfängers und die seines Kompaniechefs. Eine weitere Herausgabe eines Dokuments mit gleichem Inhalt erfolgte 1996 aufgrund einer Sammelanfrage des Landgerichts Berlin zu MfS-Unterlagen, die allgemein Gewaltakte an der ehemaligen Grenze dokumentieren.

In den Prozessen um die Todesschüsse an der DDR-Grenze in der 1990er Jahren wurden insgesamt 246 Anklagen gegen ehemalige Grenzsoldaten, ihre Vorgesetzten und führende SED-Politiker erhoben. Obwohl der Justiz kein offizielles DDR-Dokument vorlag, das einen allgemeinen Schießbefehl enthält, und die Angeklagten sich auf das DDR-Recht beriefen, stellte der Bundesgerichtshof fest, die tödlichen Schüsse an der Grenze verletzten „höherrangige Rechtsprinzipien“. Ex-Grenzsoldaten mussten in zwei Fällen eine Haftstrafe antreten. In 77 Verfahren wurden die Angeklagten zwar 
verurteilt, die Haft wurde aber zur Bewährung ausgesetzt. Sechs Kommandeure der DDR-Grenztruppen erhielten 1996 wegen Totschlags bzw. versuchten Totschlags Haftstrafen. Im so genannten Honecker-Prozess wurden 1993 DDRVerteidigungsminister Kessler, sein Stellvertreter Strelitz und der frühere SEDSekretär im DDR-Bezirk Suhl, Albrecht, zu Haftstrafen verurteilt. Die Verfahren gegen SED-Chef Honecker, Stasi-Chef Mielke und DDR-Ministerpräsident Stoph wurden wegen Krankheit eingestellt. Der letzte SED-Chef Krenz wurde am 25. August 1997 - zusammen mit den SED-Politbüromitgliedern Schabowski und Kleiber vom Berliner Landgericht wegen Totschlags verurteilt. Krenz erklärte wiederholt, zuletzt am 13. August 2007 in der „BILD-Zeitung“, es habe einen Tötungsbefehl nicht gegeben. Schabowski nannte es im „Tagesspiegel“hingegen „unerheblich, ob der Schießbefehl nun in schriftlicher Form vorlag oder nicht“. Es sei „Praxis“ gewesen, dass Menschen bei Fluchtversuchen an der Grenze erschossen wurden. Das Urteil des Berliner Landgerichts wurde durch den Bundesgerichtshof, das Bundesverfassungsgericht und schließlich, einstimmig, durch die Große Kammer des Europäischen Gerichtshofes für Menschenrechte bestätigt.

An der innerdeutschen Grenze starben nach jüngsten Berechnungen bis 19891245 Menschen, davon 231 an der Berliner Mauer. Die Berliner "Arbeitsgemeinschaft 13. August" spricht dagegen von weit mehr als 1300 Opfern. Sie zählt allerdings auch Fälle ohne Fremdeinwirkungen mit, etwa Ertrunkene in der Ostsee oder Opfer von Unfällen mit Schusswaffen.

Quelle: Robert Rauh: http://www.cornelsen.de/sixcms/media.php/8/schiessbefehl_didak.doc?siteII

\section{Anhang F: Der Fragebogen}

Please think about this teaching unit on the Berlin wall again and grade the various exercises in terms of how they holistically, i.e. as a whole fit together. Please grade the activities/techniques on a scale from 1 (not helpful at all) to 5 (very helpful).

Die Bewertungen von 15 Lerner/innen sehen folgendermaßen aus: 


\begin{tabular}{|l|l|l|l|l|l|}
\hline \multicolumn{1}{|c|}{ Activities } & $\mathbf{1}$ & $\mathbf{2}$ & $\mathbf{3}$ & $\mathbf{4}$ & $\mathbf{5}$ \\
\hline $\begin{array}{l}\text { 1. Warm up (level 1-10, apple picking and looking over a } \\
\text { wall) }\end{array}$ & 3 & & 4 & 8 & \\
\hline 2. Tourist sightseeing at the Berlin wall & 1 & 2 & 3 & 7 & 2 \\
\hline $\begin{array}{l}\text { 3. Building and 'being' the wall (gesture, sound and } \\
\text { 'l'-sentence) }\end{array}$ & 1 & 3 & 6 & 4 & 1 \\
\hline $\begin{array}{l}\text { 4. Sample sentences and different ways of speaking them } \\
\text { (individual) }\end{array}$ & 3 & 3 & 3 & 5 & 1 \\
\hline $\begin{array}{l}\text { 5. Sample sentences and different ways of speaking them } \\
\text { ('interrogation' situation) }\end{array}$ & 1 & 2 & 4 & 6 & 2 \\
\hline $\begin{array}{l}\text { 6. Reconstruction of events using only the sample } \\
\text { sentences }\end{array}$ & & 2 & 5 & 8 & \\
\hline $\begin{array}{l}\text { 7. Text reading and gap filling exercise (pronouns, } \\
\text { references) }\end{array}$ & & & 2 & 7 & 6 \\
\hline 8. Building and interpreting Standbilder/Freeze-frames & 1 & 1 & 3 & 5 & 5 \\
\hline 9. Writing a role biography & & & & 2 & $\mathbf{1 3}$ \\
\hline 10. Hot seating/hot chair & 10 & 13 & 32 & 54 & 41 \\
\hline Total: & & & 2 & 2 & $\mathbf{1 1}$ \\
\hline
\end{tabular}

Ausgewählte Teilnehmer-Bemerkungen zu den offenen Fragen des Fragebogens:

In general, should drama-based activities be incorporated into second language learning?

- After I filled in the table I conclude that the individual exercises were not very special until they began to fit together. The resulting "gestalt" is what counts. I don't remember doing each of these individual items. I guess they blended together. But, there is one thing I would like to highlight which does not fit neatly into your list. I remember being very frustrated while learning French (the second time, not my high-school experience which was a "throw away") as I could only speak in short phrases and not say anything of interest. I could only speak like a simple-minded child. [...]In the role play, or whatever it is called that we just went through here is what I think was very good about the process: We could all participate because we all had enough vocabulary as long as we stayed on topic. Indeed we had a few short phrases that we each pulled out of our notes. Further, we could each ask questions "within the frame work" and expect answers from the other students. In short, we played a role in which we could actually formulate what we wanted as we were not hindered by serious knowledge gaps. We didn't need to keep shifting the substance of what we wanted to say, or ask, according to the words or structure available to us.

- Depends on the person. Much as I love theatre, I prefer and learn better with a more traditional method.

- Yes, I believe that they should be added as they add context to the language that you are learning. It is something that can be exciting and 
therefore, can break the monotony of traditional learning methods. It also allows individuals the opportunity to speak off the cuff and therefore, improve one's language skills.

Did you enjoy these activities? Yes, why? No, why not?

- Yes because for the first time, I felt connected to the issue... the wall is something that always came up in regular conversations, as a form of government oppression and political violence; it came as a debate between socialism and capitalism and there was always a disconnect. This time however, I felt a certain emotional connection I could not pretend that no this is just a charade; it almost felt real!

- Partly, yes. I enjoyed learning more about how the wall affected individuals and the history component. But, my brain freezes in a creating-on-my-feet situation, so it doesn't work for me. This definitely was an ice-breaker and, I think, upped the bonding of the class members.

- As a language-teacher, I did enjoy them very much. I think all languageclasses could benefit from having at least a little bit of this theory built into their structures. They allow for creative use and application of the learned language, and creativity is something that I have found difficult to facilitate in the classroom-setting.

- Warm up, sightseeing, and 'being' the wall were not enjoyable, because I didn't feel they added to me understanding of the unit or to my speaking ability. The role biography and hot chair exercises stretched my abilities and made me think by putting me in the context of life behind the wall.

Do you think that learning with all senses helps you personally to learn better?

- I found it to be an innovative approach which requires familiarity in relation to the traditional German language learning courses that I attended in the past. Perhaps, I have to get a better grasp of this learning method, especially to be more readily expressive of my thoughts.

- Yes and no, it was a great experience but also somewhat emotionally overwhelming which I find compromises my ability to learn.

- I enjoyed most of the activities, but I'm a bit shy so some of the activities made me a bit uncomfortable. But, I do think that the more students are exposed to this type of teaching, the more comfortable they are with it. 


\section{Anhang G: Drei Rollenbiographien}

Rollenbiographie Gerhard - Was für ein trauriges Leben. Bin ich tot? .....Na ja klar. Ich hoffe, dass ich allerwenigstens im Himmel bin. Es ist ganz ruhig hier und fast jeder trägt weiße Kleidung. Wenigsten bin ich im richtigen Ort. Na da bin ich ja richtig froh. Übrings bin ich der Gerhard und alles, was du über mein kurzes Leben wissen musst, - kann man darunter lesen.

Mein voller Name ist Gerhard Prüss und ich bin 20 Jahre alt. Ich bin in einem kleinen Dorf in der Nähe von Cottbus geboren. Kennen Sie Drebkau? Jemand hat mir gesagt, dass die 725-Jahr-Feier der Stadt Drebkau am 2005 stattfinden wird. Ich setze drauf, dass Sie das gar nicht wussten. Meine Eltern haben sich getrennt und ich bin mit meiner Mutter im Ost-Berliner Stadtbezirk Pankow aufgewachsen. Mein Vater lebt noch in Drebkau. Ich lebte auf einer ganz normalen Ost-Berliner Straße in einem ganz normalen Ost-Berliner Plattenbau. Ich hatte eine ganz normale Kindheit mit viel Liebe von meiner Mutter und selbstverständlich von der DDR.

Wie sehe ich eigentlich aus? Ich war groß, ungefähr 1,80 Meter hoch mit einem schönen Lächeln immer auf meinem Gesicht. Ich würde sagen, dass ich leichtlebig war. Meine Freunde haben oft mir gesagt, dass ich wie Elvis in seinen guten Tagen ausgesehen habe. Ausgerechnet spielte ich die Gitarre in einer Rockabilly-Band. Wir klangen wie die Jungs von den Stray Cats. Meine Freunde haben uns komisch gefunden, weil sie lieber die Bands wie Schleim-Keim oder die Skeptiker mochten. Meine Lieblingsfußballmannschaft war Union Berlin und ich würde das Lied „Eisern Union, schlagt die Bullen“ singen, jedes mal wenn sie gegan BFC Dynamo Berlin spielten. Ich hatte mein Abitur gemacht und ich wollte gerne zur Uni gehen.

Ich war kein politischer Kerl. Ich habe mich nicht für Politik interessiert. Einige meiner besten Freunde beteiligten sich an Demonstrationen gegen den Staat oder die Gesellschaft aber meine Mutter wollte nie, dass ich mich beteiligte. Meine Oma hatte immer Angst vor den Staatssicherheitsdienst der DDR. Sie sagte immer, man weiß nie, wer ist wer. Manchmal dachte ich, war sie immer noch verärgert mit meinem Vater. Selbstverständlich wusste ich, dass mein Leben auf der anderen Seite der Mauer besser sein könnte aber im Großen und Ganzen, war ich glücklich. Mein Leben war ziemlich einfach, bevor ich meine Freundin kennengelernt habe.

Natürlich meine kurze Lebensgeschichte geht auch um Ulrike Dutschke. Was für ein schönes Mädchen. Ulrike das Mädchen meiner Träume. Ich habe sie während eines Konzerts am Weißen See von Dean Reed, den wohlbekannten amerikanischen Sozialist, kennengelernt. Das erste Mal, das ich sie sah, trug sie einen schwarzen kurzen Rock. Sie war wie ein Engel vom Himmel mit langen blonden Haaren und tiefblauen Augen. Ich hätte nie gedacht, dass sie die einzige für mich wäre. Ich muss ja einen tollen Eindruck auf sie gemacht haben. Zwei Wochen später sind wir ein Liebespaar geworden. Manchmal ist das Leben grausam. Es ist unglaublich, aber sie lebte in Neu Kölln auf der anderen Seite der Berliner Mauer.

Was kann man sagen, wenn man verliebt ist. Letzte Woche war ihr Geburtstag 
und sie konnte mich nicht sehen, weil sie so beschäftigt mit der Universität war. Ich habe gedacht, dass es eine gute Idee wäre, sie mit einem besonderen Geschenk zu überraschend. Ich habe schließlich einen Ring gekauft. Es sollte eigentlich so einfach sein, über die Mauer nach West-Berlin zu klettern. Mein Tod ist jetzt eine andere Statistik. Die Liebe hat keine Grenzen.

Rollenbiographie Gerhards Mutter - Meine Nachbarin hat gestern mich besucht. Sie war verwirrt. Als wir redeten, fang sie plötzlich zu weinen an. Sie war untröstlich. Für lange hat sie von ihrem Sohn Gerhard gesprochen, den ich gut kenne, aber ich habe ihn vor mehr als eine Woche nicht gesehen. Sie erklärte mir, was ist passiert. Ich weiß nicht, ob es wirklich passiert ist, aber das ist was man ihr gesagt hat, dass passiert ist. Man, das heißt die Polizei von hier und die Stasi.

Gerhard hat seiner Mutter gesagt, dass er wollte seinen Vater zu besuchen. Ich muss hier erklären, dass meine Nachbarin und ihr Mann sind vor sieben Jahre geschieden. Also abfahrt Gerhard seinen Vater zu besuchen. In Wirklichkeit hat er das nicht getan. Er ist an die Grenze gefahren und hat versucht über die Mauer zu klettern. Die Polizei hat ihn erwischt und in das Gefängnis geworfen. Als meine Nachbarrin dorthin gegangen ist, ihren Sohn zu sehen, hat man ihr gesagt, dass es unmöglich war. Man hat ihr gesagt, dass er sich erhängt hat. Dann hat man ihr die Urne gegeben. Jetzt glaube ich nicht, dass Gerhard sich erhängt hat. Aber was ist wirklich passiert? Das weiß ich nicht.

Meine Nachbarin ist hoffnunglos aber kann nichts tun. Sie hat mich gefragt ihr zur helfen, damit Gerhard eine anständige Bestattung haben kann. Ich werde ihr helfen.

Rollenbiographie: ein Grenzsoldat - Mein Name ist Hendrick Hundhauser. Ich bin 22 Jahre alt und ich bin Grenzsoldat seit vier Jahren. Unsere Aufgabe ist schwierig, aber notwendig. Wir bieten Sicherheit für unsere Bürger und eine Zukunft für unsere Kinder. Das Leben ist kompliziert. Niemand würde ein Zwei-jährigen Kind seine eigene Zukunft entscheiden lassen. Was nützt es der Geschichte und der Intelligenz, wenn unsere Leute es auslassen? Der Hundetrainer muss jeden Hund disziplinieren, damit jeder Hund in der Harmonie sein könnte. Auf die gleiche Weise müssen wir unsere Gemeinschaft so schützen. Leider gibt es einige Leute, die nur an sich selbst denken. Wer glaubt, dass unsere Gesellschaft nicht würdig ist, hat die Pflicht, sie zu verbessern. Nicht zu fliehen. Die Außenseiter im Zwinger, wer zu weit geht, müssen entsprechende Disziplin akzeptieren. Es ist dasselbe für den Bürger. Deshalb habe ich meine Pflicht getan bei dieser Gelegenheit und für das Wohl unseres Landes hinderte ich diesen Mann Gerhard davonzulaufen. Ich bedaure, dass er nicht aufhören würde, und so hatte ich zu schießen. Es war ein schneller und deshalb ein schmerzloser Tod. 